\title{
La Pucelle sur la scène littéraire et politique : le trajet Pont-à-Mousson - Londres
}

\section{Richard Hillman}

\section{(QpenEdition \\ 1 Journals}

\section{Édition électronique}

URL : http://journals.openedition.org/shakespeare/750

DOI : 10.4000/shakespeare.750

ISSN : 2271-6424

Éditeur

Société Française Shakespeare

\section{Édition imprimée}

Date de publication : 1 novembre 2005

Pagination : 131-150

ISBN : 2-9521475-1-5

\section{Référence électronique}

Richard Hillman, "La Pucelle sur la scène littéraire et politique : le trajet Pont-à-Mousson - Londres », Actes des congrès de la Société française Shakespeare [En ligne], 22 | 2005, mis en ligne le 01 janvier 2007, consulté le 02 mai 2019. URL : http://journals.openedition.org/shakespeare/750 ; DOI:

$10.4000 /$ shakespeare.750 


\section{Shakespeare et l'Europe de la Renaissance}

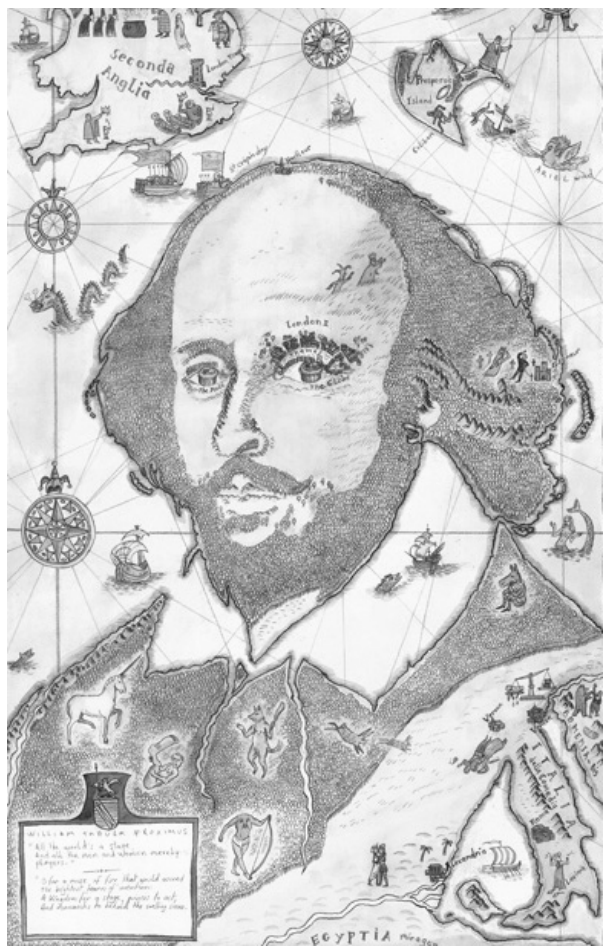

a ctes du Congrès

organisé par la

SOCIÉTÉ FRANÇAISE SHAKESPEARE

les 11,12 et 13 mars 2004

textes réunis par

Pierre KAPITANIAK

sous la direction de

Yves PEYRÉ 


\title{
COMITÉ SCIENTIFIQUE :
}

\author{
Margaret Jones-Davis \\ Jean-Marie Maguin \\ Yves Peyré \\ Pierre Kapitaniak
}

COUVERTURE :

Edouard Lekston, William Tabula Proximus, 2004

conception graphique et logo

Pierre Kapitaniak

CC 2004 Société Française Shakespeare

Institut du Monde Anglophone

Université de Paris III - Sorbonne Nouvelle

http:// univ-montp3.fr/SFS/

5 rue de l’École de Médecine

75006 Paris

ISBN 2-952 1475-1-5

Tous droits de traduction, de reproduction et d'adaptation réservés pour tous les pays 


\title{
LA Pu CELle SUR LA SCÈnE LitTÉRAire ET POLITIQUE: LE TRAJET PONT-À-MOUSSON - LONDRES
}

\author{
Richard HILLMAN
}

\begin{abstract}
Cet article précise l'idée de l'auteur, déjà présentée en ébauche dans Shakespeare, Marlowe and the Politics of France (2002), que la première tragédie française sur le sujet de Jeanne d'Arc, composée par le jésuite Fronton Du Duc à l'université de Pont-à-Mousson au printemps 1580, montée en septembre de cette année, a pu influer sur la représentation de "Joan de Pucelle " dans la première partie d'Henry VI. Certains parallèles (et même divergences) textuels confortent l'hypothèse d'un lien direct entre les deux pièces. Mais admettre qu'un dramaturge anglais a connu la pièce lorraine, publiée seulement en 1581 dans une édition dont seulement deux exemplaires existent toujours, impose l'obligation de se pencher sur les moyens de contact possibles. On peut au moins démontrer qu'un Anglais au courant de la politique anglaise actuelle, tel que Shakespeare ou Marlowe (ce dernier probablement mêlé personnellement dans des affaires politiques), aurait eu des raisons très particulières pour s'intéresser à Pont-à-Mousson. C'est un aperçu qui élargit notre sens de Shakespeare en particulier, et de ses anglais contemporains, comme "toujours déjà " impliqués dans l'Europe, à laquelle ils revenaient au niveau de l'imaginaire, tout en insistant sur leur distance, leur étrangeté, leur opposition.
\end{abstract}

La Pucelle on the Literary and Political Stage: Taking the Show on the Road from Pont-à-Mousson to London This article develops the author's idea, previously outlined in Shakespeare, Marlowe and the Politics of France (2002), that the first French tragedy on the subject of Joan of Arc, written by the Jesuit Fronton Du Duc at Pont-à-Mousson in the spring of 1580 and staged in September of that year, may have influenced the representation of "Joan de Pucelle" in 1 Henry vi. Certain parallels (and indeed divergences) support the theory of a direct link between the two plays. Yet to posit Shakespeare's familiarity with the tragedy from Lorraine, published only in a single edition (1581), of which only two copies are extant, requires considering the possible routes by which it might have come to an English dramatist's attention. It is at least possible to demonstrate that an Englishman attuned to current politics, as Shakespeare and Marlowe certainly were (the latter probably being personally involved in political affairs), would have had very particular reasons for interesting himself in Pont-à-Mousson. Such a realization broadens our sense of Shakespeare and his English contemporaries as "always already" implicated in Continental Europe, to which they continually turned imaginatively, even as they insisted on their distance, their national difference, their opposition.

la différence de certains collègues dramaturges, dont les plus
illustres sont Marlowe (The Massacre at Paris) et Chapman
(The Conspiracy and Tragedy of Charles Duke of Biron), Shakespeare n'a jamais basé une pièce de théâtre sur l'actualité européenne, si ce n'est, de façon très indirecte, Love's Labour's Lost. Ceci est d'autant plus frappant qu'une si large proportion de son œuvre concerne la politique sous plusieurs formes et surtout sous celle de l'histoire. Évidemment, il favorisait des modalités de signification plus ambiguës, plus libres et finalement plus provocatrices. Et il pouvait compter sur un public notamment alerte aux relations et ressemblances entre passé et présent, ici et ailleurs, même entre ici et nulle part, comme dans The Faerie Queene, selon un mode de penser 
capable de lire, à travers la typologie médiévale, une analyse scientifique à la Machiavel.

Pour activer cette dynamique essentiellement intertextuelle, il suffisait de troubler la structure quasi-grammaticale par laquelle une pièce soi-disant historique se constituait comme intègre, suffisante en soi pour contenir les significations qu'elle déclenchait. À l'époque, c'était la méthode la plus fréquente de mettre la fiction en rapport avec la réalité, comme en témoignent une pléthore de textes. Aussi l'histoire lointaine rejoint les années 1580 de façon retentissante lorsque, dans The Troublesome Raigne of King John (publié en 1591), la seule invasion de l'Angleterre par les Français depuis la conquête normande s'associe à une conjuration traîtresse qui se nomme «A holy knot of catholique consent», ou encore «this holy League», et se trouve secondée par un moine régicide assuré que son fait soit « meritorious ${ }^{1}$ ». Il en est de même dans Edward III, où l'auteur, que ce soit Shakespeare ou non, s'écarte brusquement de la chronique de Froissart pour introduire un certain «Duke of Lorraine », personnage sans original historique, comme l'ambassadeur de la France qui provoque et défie les Anglais à entreprendre la Guerre de cent ans dans la première scène, puis, dans la deuxième, incite le monarque écossais contre eux ${ }^{2}$.

Mes exemples ne sont pas innocents, bien entendu (si tant est qu'il puisse y en avoir). Ils indiquent mon intérêt général pour le théâtre anglais comme lieu où se jouaient des enjeux politiques à la fois historiques et actuels vis-à-vis de la France. Plus particulièrement, ils signalent l'orientation de la présente communication vers une ambassadrice lorraine plus connue qui a bel et bien défié les Anglais pour changer le cours de la Guerre de cent ans et qui, pour un public contemporain, n'était pas sans rapport avec la Sainte Ligue et même, comme j'espère le montrer, avec les troubles en Écosse par lesquels les Anglais étaient de nouveau tracassés.

Je ne répéterai pas ici ce que j'ai dit ailleurs sur le fonctionnement du personnage de «Joan de Pucelle » dans la première partie de Henry VI, pièce dont je persiste, comme Heminge et Condell,

\footnotetext{
${ }^{1}$ The Troublesome Raigne of King John, éd. Geoffrey Bullough, Narrative and Dramatic Sources of Shakespeare, vol. 4, Londres, Routledge, New York, Columbia University Press, 1962 , v. 545, 562, 925. Voir ma discussion dans Shakespeare, Marlowe and the Politics of France, Houndmills, Basingstoke, Hampshire, Palgrave, 2002, p. 58-63.

${ }^{2}$ Voir mon Shakespeare, Marlowe and the Politics of France, p. 8-9.
} 
à considérer Shakespeare comme étant l'auteur, même s'il a révisé l'œuvre d'autrui ${ }^{3}$. Je rappelle seulement que j'ai proposé une lecture de Joan comme incarnant déjà un mythe nationaliste français puissant, d'où le résultat paradoxal que les efforts des Anglais de la pièce pour la dénoncer et l'anéantir comme sorcière et femme transgressive ont pour effet ironique de ratifier son mystère, de la faire revivre au niveau imaginaire à travers l'histoire subséquente, et finalement de ratifier sa malédiction sur une Angleterre défaite en France et déchirée chez elle ${ }^{4}$. $\mathrm{Au}$ sens large, c'est une véritable mise en scène de ce qui se passe dans le texte auquel Shakespeare a puisé ce matériel. La deuxième édition des chroniques de Holinshed (1587) gonfle la rhétorique méprisante assenée à la Pucelle dans la première, tout en rajoutant, à partir de plusieurs sources françaises citées, des détails témoignant de la croissance de son mythe: en fin de compte, insérer entre parenthèses, «as their bookes make hir», n'efface pas sa désignation rapportée comme «[a] person [...] raised vp by power divine $\mathrm{e}^{5}$ ». On a beau livrer bataille sur le champ discursif: "But what puritie or regard of deuotion or conscience is in these writers trow yée, who make no consideration of hir heinous enormities, or else any difference betwéene one stirred vp by mercie diuine, or naturall loue, and a damnable sorcerer suborned by $\operatorname{satan}^{6}$ ?» L'histoire, elle, semble murmurer par la suite le dernier mot sur Jeanne prononcé par les Grandes croniques de Bretaigne, l'une des sources citées chez Holinshed, mais non guère ces paroles-ci : «Onques puis les Anglois ne prospererent en France ${ }^{7}$ ».

Mais si, chez Holinshed, l'histoire parle, pour sa part la Pucelle n'y dit aucun mot. C'est loin d'être le cas chez Shakespeare, où sa

\footnotetext{
${ }^{3}$ Le dernier assaut d'une longue série à l'idée de Shakespeare comme étant le seul auteur de l Henry VI à été donné par Gary Taylor, "Shakespeare and Others: The Authorship of Henry the Sixth, Part One », Medieval and Renaissance Drama in England, t. 7, 1995, p. 145-205. Pour un survol utile de cette question, qui d'ailleurs ne sera jamais résolue, voir la note sur le texte dans The Riverside Shakespeare, éd. G. Blakemore Evans, J. J. M. Tobin et al., $2^{\mathrm{e}}$ éd., Boston, Houghton Mifflin, 1997, p. 664 ; ce volume me sert d'édition de référence pour les œuvres de Shakespeare dans le présent article.

${ }^{4}$ Voir Shakespeare, Marlowe and the Politics of France, p. 130-52 et passim.

${ }^{5}$ Holinshed Raphael, William Harrison, et al., Holinshed's Chronicles of England, Scotland, and Ireland, éd. Henry Ellis et al., 6 vol., 1806-1807, rééd. New York, AMS, 1965, vol. 3, p. 163 .

${ }^{6}$ Holinshed et al., op. cit., vol. 3, p. 172.

${ }^{7}$ Alain Bouchart, Grandes croniques de Bretaigne, éd. Bernard Guenée et al., coll. Sources d'Histoire Médiévale Publiées par l'Institut de Recherche et d'Histoire des Textes, Paris, Centre National de la Recherche Scientifique, 1986, vol. 2, p. 309.
} 
sorcellerie unit son habilité militaire avec une éloquence capable de toucher les cœurs des hommes pour y produire ou de la foi et de l'amour - témoin Burgundy, «Either she hath bewitch'd me with her words, / Or nature makes me suddenly relent» (III.iii.58-59) - ou de la peur, comme le montre York: «Fell banning hag, enchantress, hold thy tongue » (v.iii.42). On pourrait dire que bien parler va de soi pour un personnage dramatique. C'est peut-être donc normal que cette qualité soit également mise en valeur, et d'une manière sans précédent dans les sources connues, par la seule pièce de théâtre, proprement dite, consacrée à Jeanne d'Arc avant celle de Shakespeare. (Par les termes de cette définition j'exclus sciemment Le mystère du siège d'Orléans, spectacle communal du $\mathrm{XV}^{\mathrm{e}}$ siècle, dont il ne reste qu'un manuscrit unique.) Il s'agit de L'histoire tragique de la Pucelle de Dom-Rémy, composée par le jésuite Fronton Du Duc à l'université de Pont-à-Mousson au printemps 1580. Tout le long de cette tragédie, c'est bien sûr le pouvoir de Dieu qui inspire l'éloquence de l'héroïne, de sorte qu'au lieu d'une malédiction finale («Then lead me hence; with whom I leave my curse...»[1 Henry VI, v.iv.86]), nous trouvons son image-miroir, une bénédiction prophétique, qui revient pour autant à la même chose :

Ah ! ne me pleurez point, mes amis, disait-elle,

Mais plutôt louez Dieu d'une bonne nouvelle

Que vous donne ma mort car avant que les cieux

Aient fait au soleil son cercle spatieux

Recommencer sept fois de la française terre

Vous verrez déchassé le peuple d'Angleterre.

Lors vous verrez vos chefs du dur joug allégés

Que leur grave injustice a si longtemps chargés.

(v. 2289-96) ${ }^{8}$

S'il n'y a pas d'Anglais pour dire, « hold thy tongue », cependant leur bourreau «Lui a d'un fer tordu bridé toute la bouche» (v. 2314) afin de la faire taire. Cette bouche s'est avérée aussi dangereuse que son bras, pour en juger par l'accusation lancée à son procès : «Car elle a endormi de ruse si gentille / Des princes les esprits que croire leur faisait / Etre la voix de Dieu tout ce qu'elle disait » (v. 1949-51).

Il s'agit surtout du Dauphin, qu'elle devait d'entrée de jeu persuader de sa mission divine. Tous les récits sont d'accord sur ce fait.

\footnotetext{
8 Je cite Fronton Du Duc, Lhistoire tragique de la Pucelle de Dom-Rémy, éd. Marc André Prévost, coll. Théâtre français de la Renaissance, $2^{\mathrm{e}}$ série, La tragédie à l'époque d'Henri III, vol. 2, Florence, Leo S. Olschki, Paris, Presses Universitaires de France, 2000.
} 
Mais il n'y a que deux textes de l'époque qui représentent la rencontre à Chinon sous forme de dialogue, et en l'occurrence les passages sont similaires. Voici l'échange dans 1 Henry VI:

Ask me what question thou canst possible, And I will answer unpremeditated ;

My courage but try by combat, if thou dar'st, And thou shalt find that I exceed my sex.

[...]

CHARLES. Thou hast astonish'd me with thy high terms. (I.ii.87-93)

Or, chez Fronton Du Duc, comme dans bien des récits, la preuve de la source divine des connaissances de la Pucelle est sa révélation de la prière secrète du Dauphin, sur laquelle il s'exclame,

Maintenant je connais

D'où la sagesse vient, dont tu nous étonnais,

Pour surpasser ton sexe : il serait impossible

Qu'elle cogneu cecy...

«Tu nous étonnais» / «Thou hast astonish'd me»; « surpasser ton sexe» / «exceed my sex»; «impossible» / «possible»: et ces correspondances sur quelques vers dans un dialogue entre les mêmes personnages dans des scènes correspondantes. Je laisse à des mathématiciens plus habiles que moi le calcul des probabilités, mais quitte à découvrir quelque source commune inconnue - car je crois avoir exclu tous les récits disponibles, dont aucun d'ailleurs ne prétend rapporter les mots du Dauphin - la conclusion me semble incontournable que l'auteur de la deuxième scène de 1 Henry VI connaissait la pièce antérieure ${ }^{9}$. Du moins, dorénavant, c'est à mon avis le contraire qui serait à prouver.

En fait, ce constat ne change pas grand-chose quant à ma propre lecture de chacune de ces pièces à la lumière de l'autre, ou par

\footnotetext{
${ }^{9}$ La seule autre ressemblance spécifique qui me semble mériter d'être citée comme preuve supplémentaire est entre les expressions de la détermination des Anglais au siège d'Orléans comme exprimée par Talbot chez Fronton Du Duc et le Dauphin chez Shakespeare :

Car c'est tout résolu que tout cet exercite

Demeurera plustost que de ce siège il quitte

Le fruit presque cueilli. (v. 1024-26)

Of old I know them; rather with their teeth

The walls they'll tear down than forsake the siege.

(1 Henry VI, I.ii.39-40)

À noter ici que je donne le vers 1025 de La Pucelle d'après l'édition originale de Nancy, Veuve J. Sanson, 1581 ; celle de Prévost adopte l'émendation poétique de l'éditeur du XIX ${ }^{\mathrm{e}}$ siècle, Durand de Lançon : «Mourera cent fois plustost que ce siège il quitte ».
} 
rapport à la rencontre imaginaire des dramaturges élisabéthains avec l'actualité française, voire européenne. Par le biais de l'intertextualité, j'ai déjà consacré pas mal de pages à de telles questions ${ }^{10}$, sur la prémisse que la pièce de Fronton Du Duc, commandée par le duc Charles III de Lorraine au jeune professeur de rhétorique, promouvait les intérêts de cette maison, en représentant la rédemption de la France par les armes d'un sauveur lorrain envoyé par Dieu. L'image d'une France souffrante, à cause de ses propres péchés, sous le joug des Anglais, brutaux corrupteurs de la justice et de la religion, n'était pas étrangère du tout à la propagande de croisade, de conquête par et pour la foi, par laquelle la maison de Lorraine comptait avancer ses ambitions politiques aux dépens du monarque actuel, Henri III $^{11}$. Il me semblait donc hautement significatif que la pièce dut être jouée au mois de mai 1580 devant ce dernier, supposé en route pour les eaux à Plombières, et que, cette visite ayant été annulée, officiellement pour cause de peste, le duc Charles poursuivit néanmoins ce projet, de sorte que la représentation eut lieu le 7 septembre devant un public comprenant « des princes de Lorraine les plus distingués et plusieurs généraux français ${ }^{12} »$.

Aussi, par la voie intertextuelle, La Pucelle peut lancer sa symbolique dans un espace discursif partagé par l'actualité française et, comme je l'ai indiqué, par le théâtre anglais des années 1590, très concerné par la menace sur le protestantisme et la paix représentée par la Ligue et ses alliés espagnols. Néanmoins, passer au concret, pour ainsi dire, c'est rappeler la distance géographique et culturelle considérable entre Londres et Pont-à-Mousson, ville à laquelle je n'ai pas encore trouvé une seule référence dans des documents anglais de l'époque. Admettre donc carrément qu'un dramaturge anglais a connu la pièce lorraine, éditée seulement en 1581 et dont seulement deux exemplaires existent toujours, impose l'obligation de diminuer quelque peu cette distance, même au risque de recourir à la conjecture. C'est à cet exercice que je consacrerai le reste de cet article.

\footnotetext{
${ }^{10}$ Dans Shakespeare, Marlowe and the Politics of France.

${ }^{11}$ Voir René Taveneaux, "L'Esprit de croisade en Lorraine aux XVI ${ }^{\mathrm{e}}$ et XVII ${ }^{\mathrm{e}}$ siècles », L'Europe, l'Alsace et la France, problèmes intérieurs et réactions internationales à l'époque moderne: Études réunies en l'honneur du Doyen Georges Livet pour son 70e anniversaire, Colmar, Les Éditions d'Alsace, 1986, p. 256-63.

12 « [... illustrissimi Lotharingiae principes cum proceribus et Gallicis praefectoribus non paucis» (le P. Abram, cité par Alain Cullières, «Jean Barnet, éditeur de l'Histoire de la Pucelle de Dom-Remy », Bibliothèque d'Humanisme et Renaissance, t. 55, 1993, p. 46-47).
} 
En pliant, pour ainsi dire, la carte en deux, commençons en territoire, sinon neutre, du moins contesté : le royaume d'Écosse. Si Edward III invente un duc de Lorraine qui provoque le roi écossais contre les Anglais au XIVe siècle, c'est un reflet d'événements beaucoup plus récents. À partir de 1579, Henri, duc de Guise, s'impliqua dans une série de complots, avec l'appui de plus en plus important de Philippe II d'Espagne, visant l'invasion de l'Angleterre, ainsi que de l'Écosse, la libération de la reine écossaise, prisonnière en Angleterre (dont l'oncle était le grand cardinal Charles de Lorraine), et la restauration de la religion catholique dans les deux pays. Ces événements ont été bien étudiés par les historiens, surtout dans la mesure où ils débouchèrent finalement sur la conjuration connue sous le nom de Throckmorton en 1583. Toutefois, on a eu tendance à sous-estimer, dans ce contexte, les ambitions plus hautes de la maison de Lorraine sur le plan domestique, ou plus précisément français, puisque la Lorraine ne faisait pas partie du royaume de France avant le XVIII ${ }^{\mathrm{e}}$ siècle. En effet, le duc Charles III, qui parlait aux Espagnols en 1582 de se mettre en tête d'une expédition en Écosse, avec Guise ou son frère Mayenne ${ }^{13}$, avait lui-même des aspirations royales, du moins pour son fils Henri, petit-fils bien aimé de Catherine de Médicis ${ }^{14}$. La rébellion des Guise contre Henri III ne s'est déclarée qu'en 1584, après le traité de Joinville, mais elle était en préparation depuis 1579 , sinon plus tôt, ce qui met sérieusement en question, comme nous allons le voir, cette histoire généralement acceptée d'une visite royale à Pont-à-Mousson.

Il faut comprendre dans quelle mesure les projets des Guise en Écosse dépendaient du jeune roi Jacques, qui n'avait que quatorze ans en 1580 , et qu'on essayait d'influencer par tous les moyens possibles, en contrariant l'influence des protestants et des Anglais. On a bien saisi l'importance des missionnaires jésuites à l'époque, dont les activités autant politiques que religieuses suscitaient le vif intérêt des services d'espionnage anglais, sous la direction de Francis Walsingham, et provoquaient des contre-mesures d'une sévérité croissante. Or un rôle central dans cette affaire d'Écosse a été joué par le jésuite éminent

13 Calendar of State Papers, Researches in Foreign Archives - Spain : Letters and State Papers relating to English Affairs, Preserved Principally in the Archives of Simancas Elizabeth I, 1558-1603, éd. Martin A. S. Hume, 4 vol., vol. 3 - 1580-1586, 1896, rééd. Nendeln, Lichtenstein, Kraus Reprints, 1971, n 301 1, 13 décembre 1582, p. 423.

14 Voir Louis Davillé, Les prétentions de Charles III duc de Lorraine à la couronne de France, Paris, Félix Alcan, 1908. 
Edmund Hay, lui-même écossais, qui avait déjà entrepris des ambassades importantes auprès de la reine Marie en 1562 et 1567. En 1582, au moment de mettre sur pied le dernier plan des Guise, c'est lui qui apporta à Jacques une proposition majeure de leur part, comme Walsingham le savait bien par ses agents ${ }^{15}$. Une note en français, datée du mois de mai, rapporte le désir de Jacques d'aller en France, son intention de se marier avec la princesse Christine de Lorraine, fille du duc Charles, de restaurer la religion catholique en Écosse, et ainsi de suite - toutes informations, naturellement, que Jacques s'empressa de démentir ${ }^{16}$.

Malheureusement pour les Guise, leur succès à ce moment dépendait beaucoup d'un intermédiaire influent qui leur servait d'agent dès son arrivée en Écosse en 1579 , le premier mignon du roi Jacques, le Français Esmé Stuart, seigneur d'Aubigny, créé duc de Lennox. Son rôle est confirmé par un rapport rendu à l'ambassade espagnole à Paris, puis à Philippe II, par des jésuites revenant d'Écosse (l'Anglais Holt et l'Écossais Creighton) en mai $1582^{17}$. D'Aubigny devait se retirer en France hâtivement après le coup de la faction pro-anglaise, qui a kidnappé le monarque en août 1582. Mais une année après, Jacques était de nouveau libre et vraisemblablement ouvert aux arguments des Guise ; il semblait même sur le point de céder en 1584. La mission de Hay prit fin seulement en 1585, lorsque tout espoir fut perdu : au mois de mars, Jacques avait renoncé formellement à toute idée d'une «Association» avec sa mère pour régner en Écosse, alors que le parti

\footnotetext{
15 Voir Conyers Read, Mr. Secretary Walsingham and the Policy of Queen Elizabeth, 3 vol., Oxford, Clarendon Press, 1925, vol. 2, p. 177, 246, 374-86. Les jésuites anglais Watts et Holt partirent en Écosse à la fin de 1581, les Écossais Hay et Creighton au printemps 1582, d'après Conyers Read, Lord Burghley and Queen Elizabeth, New York, Alfred A. Knopf, 1960, p. 280-81.

${ }^{16}$ Voir Calendar of the State Papers relating to Scotland Preserved in the State Paper Department of Her Majesty's Public Record Office, vol. 1, The Scottish Series, of the Reign s of Henry VIII, Edward VI, Mary, Elizabeth - 1509-1598, éd. Markham John Thorpe, Londres, Longman, Brown, Green, Longmans, and Roberts, 1858, vol. 30, $\mathrm{n}^{\circ} 2^{*}(9$ mai 1582), p. 423 , and $\mathrm{n}^{\circ} 14$ (30 juillet 1582). Plus généralement, les dépêches des années 1582 1584 évoquent vivement la situation politique mouvementée autour du roi écossais et la lourde implication d'acteurs français.

${ }^{17}$ Calendar of State Papers - Spain, vol. 3 - 1580-1586, $\mathrm{n}^{\circ} 266$ (18 mai 1582), p. 371; voir aussi Read, Lord Burghley, p. 232, et Wallace MacCaffrey, Elizabeth I, Londres, Arnold, 1993, p. 207, 435.
} 
favorisant les Anglais eut le dessus de façon définitive quelques mois plus $\operatorname{tard}^{18}$.

La promotion en Écosse de la cause catholique, et plus particulièrement lorraine, par Edmund Hay est susceptible de nous intéresser parce que le poste précédent du jésuite était le rectorat de l'Université de Pont-à-Mousson, poste qu'il tenait depuis la fondation de cette institution en 1574 jusqu'en 1581. C'était donc directement sous ses auspices que Fronton Du Duc a composé et monté La Pucelle, ainsi que sa seule autre œuvre dramatique connue (mais perdue), une tragédie de Julien l'Apostat en 1579 ou 1580 - encore un thème applicable, et certainement appliqué ailleurs, à la propagande politicoreligieuse. En partant pour l'Écosse, Hay mit-il un exemplaire ou deux de La Pucelle, nouvellement imprimée, dans ses bagages?

De toute façon, la carrière de ce personnage nous indique pourquoi le gouvernement anglais se serait intéressé à cette curieuse institution qu'était l'université lorraine. Hay en fut nommé recteur par les deux fondateurs, le cardinal Charles de Lorraine, et le duc Charles III lui-même, qui dès le début envisageaient pour l'institution un rôle activiste et orienté surtout vers l'Écosse. La reine Marie Stuart favorisa ce projet plus ou moins ouvertement dès 1581, quand elle y établit, en association avec le Pape, un séminaire particulier pour Écossais et Irlandais, dont le but était de « reconquérir l'Écosse à la foi catholique ${ }^{19} »$. Voici les racines de l'université d'après son historien, l'Abbé Eugène Martin :

La reine d'Écosse, en effet, inquiète des progrès de la réforme dans ses États et dans toute l'Angleterre, avait songé à créer sur le continent plusieurs séminaires où des apôtres et des missionnaires indigènes se prépareraient à lutter plus tard contre l'hérésie, dans leur propre pays. Or, l'Université mussipontaine avait été créée par son oncle, dans ce but spécial: elle comptait parmi ses professeurs des Écossais et des Irlandais, les PP Hay, Gordon, Oswald, Guillaume Barclay. [...]; elle semblait donc, malgré son éloignement, désignée à l'attention de la

\footnotetext{
${ }^{18}$ Sur ces événéments en Écosse, voir MacCaffrey, op. cit., p. 341, 435, et Antonia Fraser, Mary, Queen of Scots, Frogmore, St. Albans, Herts., Panther, 1970, p. 540-45.

${ }^{19}$ Pierre Lallemand, «Les Fondations monastiques à Pont-à-Mousson au temps de la Réforme catholique ", L'Université de Pont-à-Mousson et les problèmes de son temps : Actes du colloque organisé par l'Institut de Recherche Régionale en Sciences Sociales, Humaines et Économique de l'Université de Nancy II (Nancy 16-19 octobre 1972), Nancy, Université de Nancy II, 1974, p. 137; voir aussi Eugène Martin, L'Université à Pont-àMousson (1572-1768), Paris et Nancy, Berger-Levrault, 1891, p. 45. Le séminaire écossais à Pont-à-Mousson diminua en importance après la mort du Pape Grégoire III (1585) et de Marie Stuart (1587); l'an 1591 vit son transfert à Douai (Lallemand, op. cit., p. 138).
} 
nièce des Guise ; aussi Pont-à-Mousson fut-il pour quelques années, avec Douai et Reims, le lieu de refuge des îles Britanniques. Cet établissement eut tant de succès que les prêtres séculiers qui dirigeaient le séminaire anglais de Reims, envoyèrent un grand nombre de leurs sujets y perfectionner leurs études de belles-lettres et de théologie ${ }^{20}$.

Si on prend «théologie » au sens large, La Pucelle semblerait l'exercice idéal pour contribuer à un tel perfectionnement. Et étant donné le nombre d'Écossais et d'Anglais inscrits à Pont-à-Mousson ${ }^{21}$, si Fronton Du Duc cherchait des accents authentiques pour jouer les méchants, il disposait de pas mal d'acteurs convenables. (Quant à Jeanne même, on ne peut s'empêcher de se demander si ce rôle n'était pas attribué au fils du duc Charles, qui avait presque le même âge que l'héroïne, dix-sept ans, lorsqu'elle quitta la Lorraine pour sauver la France.)

Il serait surprenant si Walsingham n'avait pas ses agents à Pont-à-Mousson, comme il en avait à Reims, dont l'université était encore une fondation du cardinal de Lorraine (aussi archevêque de Reims), et où le collège anglais autrefois à Douai a été transféré en 1578 sous les auspices du duc de Guise, gouverneur de Champagne. De toute évidence, les services secrets anglais étaient parfaitement au courant des événements politiques qui se passaient à côté à Nancy. Même en 1579, Poulet, l'agent de Walsingham, a rapporté depuis Paris l'histoire racontée par un Écossais d'un projet d'invasion afin de libérer Marie Stuart. L'homme précisait «that his instructions directed him to the Ambassador of Scotland, whome he found gone to the baths in Lorraine, and to the Duke of Guise 22 .

\footnotetext{
${ }^{20}$ Martin, op. cit., p. 45. Bien que Michel Pernot (« Le Cardinal de Lorraine et la fondation de l'université de Pont-à-Mousson ", L'Université de Pont-à-Mousson et les problèmes de son tem ps, p. 52, n. 1) ne croie pas que l'orientation écossaise et politique de l'institution ait figuré dans le concept original du cardinal de Lorraine, il fait remarquer que ce dernier impliquait des Écossais dans le projet dès le début, même en dehors de Hay, et qu'une telle orientation se mit en place par la suite. Il est clair, d'après l'étude de Pernot, que le cardinal exerça toute son influence considérable pour faire réaliser le projet rapidement. D'ailleurs, les intentions politiques de son neveu Henri, duc de Guise, qui promouvait l'établissement d'une autre institution jésuite sur la frontière entre la Picardie et la Normandie en 1579 sont explicites: Guise précise l'avantage du passage en Angleterre en douze heures ou moins (voir Marc Venard, "Y a-t-il une «stratégie scolaire» des jésuites en France au XVI ${ }^{\mathrm{e}}$ siècle? ", L'Université de Pont-à-Mousson et les problèmes de Son Temps, p. 81 ).

${ }^{21}$ D'après Martin (op. cit., p. 81), en 1581 et les trois années suivantes, il y avait environ trente Écossais, le même nombre d'Anglais et d'Allemands, et six ou sept Irlandais.

22 Calendar of State Papers, Foreign Series, of the Reign of Elizabeth, Preserved in the Public Record Office, éd. Arthur John Butler, Londres, H.M.S.O., 1903, 1578-1579, n 686, p. 517. L'ambassadeur de Marie Stuart en France était un ecclésiastique catholique, James Beaton, archevêque de Glasgow.
} 
Au Carnaval, du 11 au 18 février 1580, dans une atmosphère de grande pompe et festivité, le duc Charles III tint ce que les historiens appellent la première assemblée de $\mathrm{Nancy}^{23}$. Notamment présent, avec grand train, était le duc Jean-Casimir de Bavière, figure d'une grande importance stratégique dont la reine Élisabeth venait de commander une armée mercenaire contre les Espagnols aux Pays-Bas ${ }^{24}$. Ses forces comportaient les rêttres qui avaient aidé François d'Anjou et les Huguenots dans la guerre civile récente. Le roi Henri avait accepté d'en payer la facture, mais il ne l'avait pas encore fait; le duc avait l'air impatient. Présent aussi était le chancelier de François d'Anjou, alors en négociations à la fois pour la main de la reine anglaise et pour la souveraineté des Pays-Bas ${ }^{25}$. Il y avait aussi quelques nobles protestants rebelles contre Henri, qui avaient fuit la Normandie et s'étaient réfugiés en Lorraine, ainsi que le duc de Mayenne (qui représentait Guise) et plusieurs militaires notables. Apparemment, on se mit d'accord sur un plan pour s'emparer de la ville de Strasbourg, puis entreprendre des actions jointes complexes et ambitieuses afin de déposer le roi et d'installer la Maison de Lorraine sur le trône de France. Les détails restent forcément flous, mais Jean-Marie Constant peut conclure au moins «qu'une action de grande envergure se préparait qui visait Élisabeth, les Pays-Bas et Henri III ${ }^{26}{ }^{2}$. C'est essentiellement le programme de la Ligue «officielle» quatre ans après, sauf qu'à ce moment, l'aide des protestants était toujours la bienvenue, du moins à court terme.

Sur ces affaires les Anglais avait des renseignements presque instantanés, à en juger par les deux dépêches existantes. La première, originaire de Strasbourg et datée du 16 février, avertit William Cecil, Lord Burghley, de la menace posée par la concertation de Jean-Casimir avec le duc de Mayenne à Nancy. Puis, le 23 févier une lettre de Paris informa les secrétaires à Londres non seulement que fêter Carnaval servait de prétexte pour faire de la politique, mais que «Casimir was moved by the Dukes of Lorraine and «Demayne » to associate himself

\footnotetext{
${ }^{23}$ Voir la discussion détaillée de Davillé, op. cit., p. 24-32, ainsi que Histoire et dictionnaire des Guerres de Religion, éd. Arlette Jouanna, Jacqueline Boucher, et al., Paris, Robert Laffont, 1998, p. 310.

24 MacCaffrey, op. cit. p. 193-94.

${ }^{25}$ Cette dernière négociation ayant porté fruit, le contrat fut signé au mois de septembre 1580 .

26 Jean-Marie Constant, Les Guise, Paris, Hachette, 1984, p. 200.
} 
with the House of Guise, and to take the protection of the estates of France and by force to cause some reformation ${ }^{27} »$. Plusieurs «gentlemen of Normandy» sont nommés comme participants. $\mathrm{Au}$ printemps, d'autres détails se firent connaître «by letters from those parts », tandis que le 3 avril Walsingham fut informé, encore depuis Paris, que le duc de Mayenne avait « declared to Casimir in his brother the Duke of Guise's name that France was so ill-governed that unless some remedy was found in time it might run to ruin ${ }^{28} »$; si Casimir prêtait son appui, Guise promettrait de laisser les Huguenots en paix.

Les fêtes organisées par le duc Charles autour du Carnaval comprenaient-elles une pièce de théâtre, comme pourrait laisser sousentendre son penchant connu ? Si oui, elle aurait bien pu être Julien l’Apostat, sorte de répétition pour l'événement principal que devrait être la représentation devant le visiteur royal, en route vers ces fameux bains de Lorraine, d'une croisade lorraine à la rescousse de la France. À quelle réception le roi aurait-il pu s'attendre ? Louis Davillé, se basant sur des lettres de Catherine de Médicis, évoque toute une région, y compris la Champagne, également dominée par les Guise, dans un état d'agitation, traversée de peurs de révolte de la part des protestants, ainsi que des catholiques, et surtout craintive de leur action jointe contre la monarchie ${ }^{29}$. La turbulence se fait sentir dans plusieurs documents écrits sur place, qui font référence constamment aux tensions religieuses et aux déprédations causées par des hommes armés ${ }^{30}$. Une lettre révélatrice a été gribouillée en hâte par le duc de Guise à Joachim de Dinteville, depuis peu nommé lieutenantgouverneur pour la Champagne. Datée du 27 avril 1580 - donc presque immédiatement avant la visite royale annoncée - cette dépêche confirme que la peste qui attendait Henri n'avait pas grand-chose à voir avec des microbes :

Monsieur de Dinteville, je vous renvoie ce porteur, aiant le Roy retardé son voyage pour les remuemens qui sont survenuy, et sommes icy le bec dans l'eau entre paix et guerre. L'on estime que, dans trois jours, l'on y

${ }^{27}$ Calendar of State Papers, 1579-1580, $\mathrm{n}^{\circ}$ 178. Un an plus tard, les Anglais furent inquiets au sujet d'une autre assemblée à Nancy; voir Calendar of State Papers (janvier 1581-avril $1582), \mathrm{n}^{\circ} 16$ (13 janvier 1581), p. 19, et n ${ }^{\circ} 62$ (20 février 1581), p. 68.

${ }^{28}$ Calendar of State Papers, 1579-1580, $\mathrm{n}^{\circ} 247$.

${ }^{29}$ Voir Davillé, op. cit., p. 24-25.

${ }^{30}$ Voir Georges Hérelle (éd.), La Réforme et la Ligue en Champagne, documents, 2 vol., Paris, Champion, 1887-1892. 
verra cler. Je ne fauldray vous en mander ce qui en sera. Cependant, tenez toujours noz villes adverties plus que jamais ${ }^{31}$.

Se sentant avec raison coincé entre son maître royal et le pair presque tout-puissant, Dinteville aurait-il vraiment cru en l'expression des meilleurs sentiments de son correspondant: «Votre entièrement meilleur amy à jamais. Henry de Lorraine»? Mais étant donné la situation politique, est-ce raisonnable de supposer que le roi, qui après tout n'était pas sot, entreprendrait une telle excursion pour sa santé ? Tout historien semble avoir accepté la version officielle de la visite proposée et de son annulation, mais les Anglais, eux, n'étaient pas dupes, à en juger par une dépêche de Paris à Londres du 8 avril :

Since the Queen Mother's departure - who set out on the 6th inst. towards her son at Angiers - the King has bestirred himself in seeking to appease the troubles that were arising in his realm. He entertains the «opinion» of his journey towards the «Baynes of Plumiers» [Plombières] in Loraine, seeming to await the return of his physician, Dr Miron. The other day Colonel «Chamberg ${ }^{32}$ » was despatched by him to Germany under colour of visiting the frontiers, there, and a place fit for the king's lodging; but in fact rather to understand Casimir's dealing with certain Norman gentlemen who are there ${ }^{33}$.

Voilà, en somme, deux routes qui, bien que passant respectivement par l'Écosse et par Paris, diminuent la distance imaginaire entre Pont-à-Mousson et Londres. Pour ce qui est de Shakespeare personnellement, on est, comme d'habitude, dans le flou. On pourrait au moins évoquer, emboîtant le pas à Charles Nicholl sur la piste de Marlowe ${ }^{34}$, le chevauchement remarquable dans les années 1580 et 1590 entre la communauté littéraire londonienne et le demimonde complexe et indistinct d'espions, contre-espions et leurs connaissances, dont les activités portaient surtout sur les Récusants. On pourrait y rajouter le fait connu que l'ambassadeur anglais à Paris était chargé de fournir à Walsingham les copies de textes susceptibles de nuire aux intérêts nationaux ${ }^{35}$. Toutefois, je cède à la tentation de

\footnotetext{
31 Cité dans Hérelle, op. cit., vol. 2, p. 102-3.

32 C'est-à-dire, Gaspard de Schomberg, comte de Nanteuil.

33 Calendar of State Papers (1579-1580), $\mathrm{n}^{\circ} 252$, p. 217.

34 Dans The Reckoning : The Murder of Christopher Marlowe, Londres, Jonathan Cape, 1992.

35 Voir Lisa Ferraro Parmelee, Good Newes from Fraunce: French Anti-League Propaganda in Late Elizabethan England, Rochester, NY, University of Rochester Press, 1996, p. 38 .
} 
replier la carte, mais de l'autre côté cette fois, pour rapprocher Édimbourg de Londres, et peut-être même de Stratford-upon-Avon.

Le Calendar of State Papers, Scottish Series, contient une plainte, datée du mois d'avril 1583 - donc juste au moment où la lutte en Écosse entre protestants et catholiques était la plus intense, avec le jeune roi au milieu - comme quoi un certain Vautrollier, «a Frenchman », se heurte à un harcèlement dans ses efforts pour établir une imprimerie en Écosse, malgré la détention d'un permis ${ }^{36}$. Il s'agit bien évidemment de Thomas Vautrollier, l'imprimeur huguenot bien connu, basé à Londres, qui était notamment actif dans l'édition d'ouvrages liés à la France, y compris des textes d'orientation hautement politique, tels que la traduction par Arthur Golding de la Vita hagiographique de l'amiral Coligny ${ }^{37}$. Les grandes lignes de ses activités écossaises sont connues : il avait été encouragé à s'y établir en tant que «stranger banished for religion » qui offrait « to imploy his labour [...] for the weill of the country», et il y aurait passé deux séjours de deux ans chacun (1580-1582 et 1584-1586), en faisant bonne affaire et en vendant des livres au roi $^{38}$. Or il est possible, avec une certaine confiance, de lire entre ces lignes, étant donné la turbulence politico-religieuse en Écosse à l'époque et le fait qu'en 1584, c'était à l'imprimerie londonienne de Vautrollier que Burghley confia les versions en français et en latin de son traité anonyme justifiant l'exécution du jésuite Edmund Campion ${ }^{39}$. En toute probabilité,

\footnotetext{
36 Calendar of State Papers relating to Scotland, vol. 1 (1509-1598), vol. 31 (1583), n ${ }^{\circ} 93$ (9 avril).

${ }^{37}$ The lyfe of the most godly, valeant and noble capteine and maintener of the trew Christian religion in Fraunce, Iasper Colignie Shatilion, sometyme greate admirall of Fraunce, trad. Arthur Golding, London, Thomas Vautrollier, 1576 ; sTC 22248. Cet ouvrage très influent est attribué à Jean de Serres, à Jean Hotman ou encore à François Hotman. L'attribution à ce dernier est maintenue de façon convaincante par l'éditeur scientifique dans François Hotman, La vie de Messire Gaspar de Colligny Admiral de France (c. 1577), éd. Émile-V. Telle, 1643, rééd. en fac-similé, Genève, Droz, 1987, p. 99-100.

38 A. E. M. Kirwood, «Richard Field, Printer, 1589-1624 », The Library, t. 12, n 1, 1931, p. 3-4.

${ }^{39}$ The Execution of Justice in England, not for Religion but for Treason, Londres, C. Barker, 1583 ; STC 4902. Les traductions en français et en latin sont, respectivement, STC 4906 et 4904 . Cette campagne de publication était très efficace, incluant, toujours en 1583, des traductions en néerlandais (publiée à Middelburg) et en italien, cette dernière attribuée à G[iovanni] Wolfio, i.e., John Wolfe, autre éditeur londonien actif dans la cause huguenote et plus largement protestante; voir Denis B. Woodfield, Surreptitious Printing in England, 1550-1650, New York, Bibliographical Society of America, 1973, p. 24-33, en particulier p. 25, ainsi que mon livre Shakespeare, Marlowe and the Politics of France, passim). Sur les contextes politiques de cet ouvrage, voir Read, Lord Burghley, p. 244-55.
} 
l'entreprise écossaise fut commanditée par le gouvernement anglais, avec la complicité de leurs alliés sur le terrain, pour fournir de la propagande contre celle importée de France, surtout par les jésuites. Le harcèlement que Vautrollier était censé avoir subi en 1583 dut le forcer à quitter temporairement le pays.

Les années d'activité de Vautrollier en Écosse ont laissé relativement peu de traces identifiables, ce qui, avec les quelques produits documentés, conforte l'hypothèse d'un engagement dans la propagande éphémère ${ }^{40}$. Ne sont documentées qu'une poignée d'ouvrages d'orientation moralisatrice et religieuse, ardemment protestants, ainsi que les deux premières traductions anglaises du poète, diplomate et guerrier huguenot, Guillaume de Salluste, seigneur Du Bartas. Le fait qu'une de ces traductions était du roi Jacques luimême semble signaler un effort d'encourager le roi dans cette sympathie particulière avec le poète qui aboutit à la visite écossaise de ce dernier en 1587, visite qui visait probablement une fin politique distincte $^{41}$.

Cependant c'est l'autre traduction qui s'avère plus intrigante. Il s'agit de La Judit, exaltation du personnage biblique parue premièrement en 1574, et traduite en 1584 par Thomas Hudson, musicien du ménage royal. Dans son Épître dédicatoire, Hudson présente son travail non seulement comme une commande royale de composer sur «an agreable Subiect to your highness», mais même comme une sorte de collaboration avec le roi, qui l'aida avec les

\footnotetext{
${ }^{40}$ Il est tentant, vu la date, la langue et l'orientation politique, d'attribuer à Vautrollier un pamphlet de 1582 : Remonstrance faicte au Roy d'Escosse par Messieurs de son Conseil privé conjoinctz avec la Noblesse d'iceluy pays, sur les practiques d'Aubigny, au moys d'Octobre. Traduict d'Escossois en François. Le catalogue de la British Library propose Paris comme son lieu de publication (toutefois avec un point d'interrogation), et l'ouvrage ne figure pas comme produit britannique dans le Short Title Catalogue. Par contre, le catalogue de la Bibliothèque Nationale de France n'offre pas de conjecture en ce qui concerne le lieu, alors que les circonstances confortent l'hypothèse d'une origine écossaise. Une analyse bibliographique matérielle pourrait être utile.

${ }^{41} \mathrm{La}$ traduction était de l'Uranie, ou Muse celeste, dans The essayes of a prentise, in the diuine art of poesie, Édimbourg, T. Vautrollier, 1584, STC 14373, avec une seconde édition l'année suivante (STC 14374). Du Bartas se rendit en Écosse entre mai et septembre 1587; vraisemblablement il fut chargé par Henri de Navarre de promouvoir un mariage entre sa sœur Catherine et le roi Jacques; le poète passa par l'Angleterre, où il participa peut-être à des discussions préalables. Voir Harry Ashton, Du Bartas en Angleterre, 1908, rééd. Genève, Slatkine, 1969, p. 18-30 ; Urban Tigner Holmes, Jr., Guillaume De Salluste Sieur Du Bartas: A Biographical and Critical Study, Chapel Hill, University of North Carolina Press, 1935, p. 21-22 ; et James Dauphiné, «Le 'chevalier' Du Bartas: lettre inédite de Jacques VI d'Écosse", Bibliothèque d'Humanisme et Renaissance, t. 59, n 1, 1997, p. 63 66.
} 
révisions et la correction ${ }^{42}$. La matière liminaire unit les compliments habituels au monarque (visant surtout son goût littéraire) à un discours résolument protestant, et le traducteur interpole des vers impliquant le roi Jacques dans le but sacré visé par le poème,

... since in vulgar verse I prease to sing

This godly Poome to a Christian King,

To him who God in goodnesse hath erect

For princely Piller, to his ow ne elect :

For law full Lord, to raigne with trueth and right...

$\left(\right.$ sig. $\left.\mathrm{B} \mathrm{r}^{\circ}\right)$

Dans ce contexte, on peut considérer que le but du poème bartésien était loin d'être purement spirituel.

En effet, La Judit occupait une place très particulière dans la propagande huguenote, dans la mesure où le poème donnait voix à la lecture providentielle de l'assassinat en 1563 de François, duc de Guise, père du duc actuel ${ }^{43}$. Il venait d'être réédité (en 1582 et 1583 ) avec des commentaires calvinistes tranchants de Simon Goulart, ce qui accrut son prestige et sa force comme document protestant militant. François, duc de Guise, fut abattu pendant son siège d'Orléans, ville alors tenue par les protestants, par un gentilhomme français terre-à-terre (et, d'après les Guise et autres, incité par Coligny), mais on représentait sa mort comme la délivrance miraculeuse du peuple élu de Dieu. L’image de Guise est assimilée à celle d'Holoferne, tyran blasphématoire à la tête d'une armée étrangère (avec allusion aux forces espagnoles et allemandes qui appuyaient les catholiques). Comme l'acte héroïque d'une faible femme, infusée de force divine, avait permis de lever le siège de Béthulie, l'exécution de Guise sauvait Orléans.

Mais il y avait eu, bien sûr, un siège d'Orléans précédent, et d'après la pensée typologique de l'époque, mettre les deux sièges en

\footnotetext{
42 Guillaume de Salluste, seigneur Du Bartas, The historie of Iudith in forme of a poeme. Penned in French, by the noble poet, G. Salust. Lord of Bartas, trad. Thomas Hudson, Edinburgh, Thomas Vautrollier, 1584, STC 21671, sig. Aiii $\mathrm{r}^{\circ}-\mathrm{v}^{\circ}$. Il y a une ambiguïté intrigante quant au public visé par ce volume : d'une part, non seulement celui-ci est dédié au roi Jacques, mais il revendique une intimité et une sympathie intellectuelles avec lui ; d'autre part, il contient des notes explicatives destinées à un lectorat peu érudit (donc glosant des personnages tels que «Abraham », «Agamemnon » ou encore « Mars»). On a l'impression d'une tentative de promouvoir une solidarité spécifiquement protestante entre le roi et ses sujets.

${ }^{43}$ Sur le symbolisme politique du poème, voir Guillaume de Salluste, sieur Du Bartas, $L a$ Judit, éd. André Baïche, Publications de la Faculté des Lettres et Sciences Humaines de Toulouse, sér. A, t. 2, Toulouse, Association des Publications de la Faculté des Lettres et Sciences Humaines de Toulouse, 1971, p. xxi-cixc.
} 
parallèle, ou plutôt en contraste, était de rigueur: en 1576, les conseillers municipaux d'une Orléans résolument retournée au catholicisme (avec le renfort de Catherine de Médicis, qui avait doté d'un morceau de la vraie croix la cathédrale saccagée par les protestants) ont commandé la publication d'une histoire du siège de 1429 mettant Jeanne notamment en valeur ${ }^{44}$. Ce document, L'Histoire et discours au vray du siège qui fut mis devant la vile d'Orléans par les Anglais, prouve de façon décisive que l'association de Jeanne avec le siège était un enjeu autant pour les protestants que pour les catholiques. La preuve se trouve dans l'épître liminaire de l'éditeur juré d'Orléans, Saturny Hottot, ainsi que dans l'«Aduertissement av lectevr » annexé au récit. La lettre présente le volume comme une sorte de seconde réhabilitation de Jeanne, dont le miracle démontre que les Français sont «vn peuple aussi bien aymé de Dieu que furent iadis les Israëlites»; le but est de «faire reuiure la gloire de celle, les monuments de laquelle vous avez remis sus en vostre cité, abbatus par l'insolence des guerres ciuiles ${ }^{45} \gg$. La polémique partisane et les détails des dégâts faits par les protestants sont encore plus évidents dans l'«Aduertissement» annexe, qui concerne la procession annuelle en honneur de Jeanne, maintenant rétablie avec une deuxième délivrance de la ville comprise dans les remerciements offerts à Dieu. C'était le culte de Jeanne, semble-t-il, qui a provoqué les protestants à outrager « les cueurs des naturels François » pendant

nos premiers troubles, lors-que quelques soldats insolens \& insensez se ruerent de rage sur la statuë honorable de ceste chasté [sic] Amazone, Ieanne la Pucelle, qu'ils abbatirent de dessus son pilier esleué sur Loyre à Orleans, \& la briserent furieusement. (sig. $\mathrm{N}$ ii $\mathrm{v}^{\circ}$ )

Dans ce contexte bien chargé, la Judit de Du Bartas revient à une virtuelle anti-Jeanne. Rajoutons à cela le fait que Du Bartas modela sa Judith sur encore une autre Jeanne - l'héroïne protestante Jeanne d'Albret, reine de Navarre ${ }^{46}$ - et nous voyons jusqu'à quel point le poème s’inscrit sous le signe de l'iconoclasme.

\footnotetext{
${ }^{44}$ Françoise Michaud-Fréjaville, «Personne, Personnage : Jeanne d'Arc en France au XVII ${ }^{\mathrm{e}}$ siècle », Jeanne d'Arc en garde à vue, éd. Dominique Goy-Blanquet, Bruxelles, Le Cri, 1999 , p. 61 et n. 30 .

${ }^{45}$ Léon Tripault, Lhistoire et discours au vray du siège qui fut mis devant la ville d'Orléans par les Anglois... plus un echo contenant les signularitez de ladicte ville, par M. Léon Tripault, Paris, Saturny Hottot, 1576, sig. aii v .

${ }^{46}$ Baïche, op. cit., p. xxi-xxxv.
} 
L'association de Jeanne d'Arc à Judith a été instaurée assez tôt $\mathrm{t}^{47}$ et diffusée largement dès au moins 1580 , avec la première édition du Recueil des rois de France de Jean Du Tillet, ouvrage auquel l'idée est attribué, avec mépris («this tale of Tillets»), dans la seconde édition des Chronicles de Holinshed ${ }^{48}$. Mais la pièce de Fronton Du Duc insiste sur cette association de manière récurrente ${ }^{49}$, et à la lumière intertextuelle du poème de $\mathrm{Du}$ Bartas (qui vient d'être réédité en 1579), ce lien revêt un caractère agressif, sinon défensif, dans l'éloge dramatique de la Maison de Lorraine. Si La Pucelle faisait partie de la campagne de propagande en Écosse, visant surtout le jeune roi, l'édition / traduction du poème bartésien aurait servi de contre-coup assez précis. Et si Vautrollier était revenu d'un séjour en Écosse avec quelques échantillons de la production des adversaires dans ses bagages...?

Il est possible aussi que Vautrollier ait été accompagné de temps à autres de son apprenti. Or les shakespeariens savent bien que le seul apprenti de Vautrollier était Richard Field, une connaissance de Shakespeare, probablement un ami personnel, depuis leur enfance passée ensemble à Stratford-upon-Avon ${ }^{50}$. Field deviendra l'éditeur de Vénus et Adonis en 1593 et du Viol de Lucrèce en 1594 ; Shakespeare semble lui rendre un hommage affectueux dans Cymbeline (1609-10), lorsqu'Imogen s'invente un maître nommé «Richard du Champ » (IV.ii.377) $^{51}$. La forme française du nom est hautement appropriée, car lorsque Field a repris l'imprimerie à la mort de Vautrollier en 1588, en devenant aussi le mari de la veuve Jacqueline, comme le voulait la coutume, il a maintenu dans une mesure considérable l'orientation linguistique et politique de la maison.

Jean-Marie et Angela Maguin nous ont rendu service, entre autres, en attirant l'attention sur Field comme l'éditeur de quelques

\footnotetext{
${ }^{47}$ Voir Jan Joseph Soons, Jeanne d'Arc au théâtre. Étude sur la plus ancienne tragédie, suivie d'une liste chronologique des a uvres dramatiques dont Jeanne d'Arc a fourni le sujet en France de 1890 à 1926, Purmerend, J. Muusses, 1929, p. 87.

${ }^{48}$ Holinshed, op. cit., vol. 3, p. 172, dont la première mention de Jeanne, avec plusieurs sources françaises citées dans la marge, y compris Du Tillet, incorpore des détails correspondant au Recueil, 2 vol., Paris, A. Perier, 1607, vol. 1, p. 233.

49 Fronton Du Duc, op. cit., v. 712-13, 1598-1601, 2390, 2393-98.

50 Voir Jean-Marie Maguin et Angela Maguin, William Shakespeare, Paris, Fayard, 1996, p. 306-7.

${ }^{51}$ Field s'est doté du nom de Ricardo del Campo pour certains imprimés faussés d'expression espagnole (voir Woodfield, op. cit., p. 41).
} 
ouvrages importants comme textes-source de Shakespeare. Il me paraît pertinent de rappeler que Field était aussi profondément impliqué, comme l'ont démontré Denis B. Woodfield et Lisa Ferraro Parmelee, dans la production de propagande contre la Ligue, y compris des documents faussés, en association avec John Wolfe ${ }^{52}$. Les premiers produits de Field pour son propre compte (ou plus précisément dits imprimés par «J. Vautrollier for Richard Field») comprenaient encore de la propagande du service secret : les versions anglaise et française (la dernière étant probablement antérieure), chacune dans des éditions multiples, de la lettre supposée écrite a Barnardino de Mendoza par le jésuite Richard Leigh exécuté, mais en réalité contrefaite par Burghley lui-même. C'était un coup brillant - dit Read Conyers, «Of all Burghley's contributions to the literature of propaganda, this letter to Mendoza was the best thing he ever $\operatorname{did}^{53} »-$ et une opération menée à grande échelle, bien coordonnée (c'était encore John Wolfe qui était chargé de la version italienne, aux ordres de Walsingham ${ }^{54}$ ). Apparemment, Field a bien fait son apprentissage dans tous les sens ${ }^{55}$.

52 Parmelee, op. cit., p. 34-35 ; Woodfield, op. cit., p. 24-45. Wolfe, un associé de Gabriel Harvey pendant un moment, était aussi notoire, du mois tôt dans sa carrière, pour ses pratiques commerciales douteuses. Sur ces questions, see Harry R. Hoppe, «John Wolfe, Printer and Publisher, 1579-1601», The Library, 4e sér., t. 14, 1933, p. 241-88, et Clifford Chalmers Huffman, Elizabethan Impressions : John Wolfe and His Press, New York, AMS, 1988 .

53 Read, Lord Burghley, p. 433. Read explique cette campagne de propragande de façon détaillée (p. 431-33 et 580, n. 91). Voir aussi Woodfield, op. cit., p. 27-28. Les références du STC sont les suivantes : 15412-15414.6.

54 Kirwood, op. cit., p. 8.

55 Kirwood (op. cit., p. 8) admet qu'il y a peut-être «some signifiance» du fait que Vautrollier est impliqué dans le coup antérieur de Burghley contre les jésuites, Field dans celui plus tardif, mais il ne développe pas cet aperçu. Voir, par contre, Woodfield, op. cit., p. 35 .

Field semble avoir maintenu un certain lien avec l'imprimerie quasi-officielle écossaise : encore un volume de traductions de Du Bartas par le roi Jacques, publiée à Édimbourg par l'imprimeur royal R. Waldegrave en 1591, avait été inscrit au nom de Field sur le Stationers' Register en 1589. Et il reste un fait curieux que pour A Midsummer Night's Dream Shakespeare se serait servi d'une autre publication de Waldegrave aussitôt après sa parution en 1594, à savoir le récit du baptême du Prince Henry d'Écosse : A trve reportarie of the most trivmphant, and royal accomplishment of the Baptisme of the most Excellent, right High, and mightie Prince, Frederik Henry, By the grace of God, Prince of Scotland. Solemnized the 30. day of August. 1594, [Édimbourg], R. Waldegrave ["Printer to the K. Majestie»], 1594 ; sTC 11214.6. Voir William Shakespeare, A Midsummer Night's Dream, éd. Peter Holland, coll. Oxford World's Classics, Oxford, Oxford University Press, 1994, n. à I.i.67-68, et Richard Hillman, "Prince Henry's Godfather and the Succession», The Struggle for the Succession in Late Elizabethan England: Politics, Polemics and Cultural Representations, éd. Jean-Christophe Mayer, préface de Jenny Wormald, coll. « Astraea », $\mathrm{n}^{\circ}$ 11, Montpellier, Université Paul-Valéry, 2004, p. 317-30. 
Certes, on peut facilement imaginer des pistes moins exotiques par lesquelles Shakespeare, ou un collègue, aurait pu arriver à une connaissance de La Pucelle du jésuite lorrain. Mais on peut également maintenir que de tels exercices d'imagination, plus colorés, ont leur propre valeur, à condition que les pistes restent bien praticables. Cette valeur consisterait, non pas à établir des faits, mais plutôt à évoquer des contextes de significations cernant le manque de faits. Lorsqu'il s'agit des significations politiques, religieuses et littéraires, toutes étroitement imbriquées, qui circulaient à travers l'Europe de l'époque, parfois avec l'effet de redéfinir ses frontières, se donner de temps à temps à de tels jeux d'esprit ne revient pas à jouer aux cartes.

Richard HILLMAN Université François Rabelais, Tours 